\title{
Condiciones para el acceso a la vivienda subsidiada en el departamento de Caaguazú
}

\author{
Ibarrola $\mathrm{A}^{*}$ \\ * Universidad Americana, Asuncion, Paraguay.
}

Resumen. Con los nuevos procedimientos se logra una mejor convivencia, con habitantes capaces de cooperar y mantener la sostenibilidad, la armonía dentro de un marco de respeto y la forma de vivir en una zona saludable, con una mirada sustentable y sostenible de una situación, localizada en el departamento de Caaguazú, vivienda social Bonanza del barrio Balcón Ovetense de la ciudad de Coronel Oviedo. El método, consiste en un nuevo procedimiento que permita establecer estrategias y técnicas de selección y/o adjudicación en los ítems de aprobación y recepción de documentos, considerando un factor muy importante que es declarar el grado académico cursado con el aval correspondiente y con sus certificaciones pertinentes de cada familia o el titular de la vivienda en proceso. Se espera con esto, que el método y la nueva técnica establezca mecanismos de postulación, claras y transparentes en su gestión, brindando una jerarquía, en la cual, la colaboración y la sostenibilidad serán los pilares fundamentales durante el proceso de la convivencia en las viviendas sociales para la sustentabilidad. Con la discusión, se llega a la conclusión de fomentar la preparación académica de los diferentes ocupantes a fin de lograr una armonía cultural y el buen funcionamiento del entorno habitacional con la concienciación y mantenibilidad de las diferentes propuestas, servicios. Con esta estrategia de la investigación se lograra segmentar y fomentar el nivel académico de todos sus pobladores con el fin de obtener la formación y capacitación cultural adecuada con la intensión de saber sobre llevar las distintas situaciones y/o problemática que surgiere de forma sostenible por el bien común.

Palabras clave. Condiciones, Grado Académico, Sustentabilidad, Convivencia, Cultural, Vivienda.

Abstract. With the new procedures a better coexistence is achieved, with inhabitants capable of cooperating and maintaining sustainability, harmony within a framework of respect and the way of living in a healthy area, with a sustainable and sustainable look of a situation, located in the department of Caaguazú, social housing Bonanza of the Balcón Ovetense neighborhood of the city of Coronel Oviedo. The method consists of a new procedure that allows to establish strategies and techniques for selection and / or adjudication in the items of approval and receipt of documents, considering a very important factor that is to declare the academic degree taken with the corresponding endorsement and with its certifications relevant of each family or the owner of the housing in process. With this, it is expected that the method and the new technique establish postulation mechanisms, clear and transparent in its management, providing a hierarchy, in which, collaboration and sustainability will be the fundamental pillars during the process of living together in homes Social for sustainability. With the discussion, the conclusion is reached to promote the academic preparation of the different occupants in order to achieve a cultural harmony and the proper functioning of the housing environment with the awareness 
and maintainability of the different proposals, services. With this research strategy, it will be possible to segment and promote the academic level of all its inhabitants in order to obtain the appropriate cultural education and training with the intention of knowing about carrying the different situations and / or problems that arise in a sustainable way by the common benefit.

Keywords. Procedure, Academic Degree, Sustainability, Coexistence, Cultural, living place.

Introducción. El Fondo Nacional de Vivienda Social (FONAVIS) se creó en el 2009 bajo la Ley 3.637/9 para facilitar el acceso a la vivienda de las familias de escasos recursos, pertenecientes a los estratos de pobreza, extrema pobreza y clase media baja canalizando subsidios bajo un programa de vivienda social. FONAVIS tiene cuatro niveles de subsidio dependiendo de los ingresos familiares de los beneficiarios. La Ley de FONAVIS indica que se puede subsidiar hasta $15 \%$ del valor de una solución habitacional en el Nivel 1, hasta el 40\% en el Nivel 2, hasta el 70\% en el Nivel 3 y hasta el 95\% en el Nivel 4. En la práctica, FONAVIS otorga el tope de subsidios para cada Nivel, lo que reduce el número de subsidios a entregar y por lo tanto se beneficia a menos familias con subsidios unitarios más altos ( $G, 2014)$.

Los requisitos para acceder a los distintos programas no consideran una diferenciación de acuerdo a los ingresos de los postulantes, sino que son los mismos interesados quienes se ubican en uno u otro programa de acuerdo a sus aspiraciones, preferencias y disposición a pagar por una vivienda (Arrieta, 1999).

La investigación propone insertar un ítem de mucha importancia dentro de los procedimientos, a fin de dar inicio a un proceso que pueda contribuir con el acceso a la vivienda sustentable, y de esta manera dar una nuevo escenario a la vivienda social, incubando una nueva modalidad de estilo y el urbanismo popular en los distintos sectores sociales con un enfoque sustentable y que este sea modelo de un nuevo sistema de procesos en la adjudicación de las viviendas sociales.

Se pretende con esta investigación otorgar mayor visibilidad de los conocimientos adquiridos por los habitantes, la preparación y/o capacitaciones son fomentadas desde el inicio de la convivencia, por lo tanto esto deriva a una mayor armonía en el hábitat. 
Es por ello, que esta propuesta modelo propone generar nuevas alternativas e ideas de gobierno en las viviendas sociales, enfocado en un urbe popular donde los habitantes apuesten a la educación y concienciación con miras sustentables.

De este modo se pretende fomentar el acompañamiento cultural, romper el paradigma, reflejar un estilo de vida más amena, con lo cual se dé un nuevo conjunto de habitantes en donde la convivencia sea más armónico y dinámica. En mayor medida de los habitantes de estos sectores, son admitidos sin previos controles culturales, con solo demostrar los documentos legales y muchas veces plagiando o con intensiones engañosas o amenazantes en común acuerdo con los representantes de los sectores populares logran empañar la gestión y lograr sus propósitos.

En tanto la diversidad de los habitantes en los diferentes territorios sociales se ven ocupados por habitantes que su mayoría no poseen una preparación académica, y o capacitación necesaria para enfrentar los distintos escenarios de forma autónoma y eficaz.

El ítem propone la imposición y exigencia a cada habitante y/o jefe de hogar el grado académico alcanzado, documentos que avales tales especialidades que desempeñan y otros. Esta estrategia promueve el saneamiento, equilibrio, y el dinamismo armónico entre sus habitantes.

Este trabajo de investigación, utilizara la estrategia de procedimiento en la selección de la población a fin de procesar los informes de cada uno de los componentes y las atenciones requeridas para la atención correspondiente.

El propósito es mejorar la calidad de vida de los habitantes mediante criterios adaptados a fin de mantener una armonía y una mejor organización en el vivir de cada día y sobre llevar los distintos escenarios que puedas presentarse.

Este investigador propone el criterio de procedimiento a fin de obtener un mejor resultado, y buen vivir de todos sus habitantes de una manera armónica y pacífica, y que este sea un modelo del nuevo sistema de convivencia en un hábitat sustentable y sostenida.

Según el programa de la vivienda en el Paraguay estos son las condiciones y requerimientos para el acceso a la compra de la vivienda por parte del gobierno. 


\section{PROGRAMA DE VIVIENDAS DE CLASE MEDIA \\ POSTULACIÓN PARA EL SUBSIDIO SEGÚN LEY 5638-16 \\ PARA COMPRA DE PRIMERA VIVIENDA}

Las personas que cumplan con los siguientes requisitos podrán acceder al programa:

* Persona mayor de edad

* Madre/Padre soltera/o, divorciado, viudo/a.

* Pareja de casados o concubinos con o sin hijos.

* Hijo/a Sostén

\section{Sus grupos familiares estarán constituidos por las siguientes personas:}

- Su cónyuge o concubino/a; los hijos menores de edad no emancipados del/la postulante, de su conyugue o concubino/a que habiten con ellos, incluidos aquellos que cumplan con la mayoría de edad durante el año calendario en que se postula;

- Los hijos del/la postulante, de su conyugue o concubino/a, con algún tipo de discapacidad sean menores o mayores de edad y que habiten con ellos;

- Las personas respecto de las cuales el/la postulante, su cónyuge o concubino/a, tenga a su cargo la tutela o curatela legal, o la obligación de prestar alimentos de acuerdo a la legislación civil y que residan con ellos.

- $\mathrm{Su}$ padre y/o madre que conforman un único grupo familiar, hijo/a mayor de edad que conforman un único grupo familiar.

\section{Requisitos básicos que deben reunir los postulantes:}

a. Nacionalidad paraguaya de un postulante o de un miembro de su familia

b. Postulante extranjero con radicación en el país y con al menos un miembro del grupo familiar con nacionalidad paraguaya

c. Postulante mayor de edad en ejercicio pleno de sus derechos civiles.

d. Postulante jefe de familia constituida por matrimonio, unión de hecho o hijo/a sostén. 
e. No ser propietario ni hallarse en trámite de compra de un inmueble, tanto el postulante como los demás miembro del grupo familiar.

f. No haber adquirido, construido, ampliado o mejorado una vivienda con la aplicación de un subsidio o ayuda estatal, o municipal, otorgada por cualquier institución publica o municipal.

g. No contar con operaciones morosas, demandas e inhibiciones.

h. Ingreso familiar de 2 (dos) salarios mínimos

i. Haber presentado toda la documentación requerida.

Viudo/a: Acta de defunción del cónyuge

Hijo/a sostén: Certificado de hijo/a sostén expedido por el juzgado de la jurisdicción donde pertenece el/la postulante; certificado de nacimiento del solicitante que compruebe la relación parental entre el mismo/a y el/los padre/s.

Padre soltero: Tenencia legal del/los hijo/s expedida por el Poder Judicial.

4. Certificado de nacimiento original de los hijos.

5. Certificado de antecedente policial original del postulante, su cónyuge o concubino/a y demás miembros mayores de edad que aporten a la economía familiar. 6. Certificado de antecedente judicial original del postulante, su cónyuge o concubino/a y demás miembros mayores de edad que aporten a la economía familiar.

7. Certificado de trabajo original del postulante, y los ingresos justificados de su cónyuge o concubino/a, además del de los miembros mayores de edad del grupo familiar que aporten la economía familiar (en caso de ser asalariados); que incluya los datos del monto percibido, antigüedad en la organización y los datos de contacto de la empresa o institución.

8. Liquidación de salario de los últimos 6 meses Contribuyentes de IVA:

- Certificado de cumplimiento tributario emitido por SET

- DDJJ de IVA de los últimos seis meses

- Copia de RUC

- Copia de formulario de inscripción del RUC, del postulante, su cónyuge o concubino/a y demás miembros del grupo familiar.

9. No contribuyentes de IVA: certificado de no ser contribuyente emitido por la SET $\mathbf{1 0 .}$ Certificado de no poseer inmuebles expedido por la Dirección General de los 


\section{Documentación requerida}

1. Formulario de inscripción. (Disponible en www.muvh.gov.py y en la oficinas del Muvh)

2. Fotocopia autenticada de la Cedula de identidad vigente y/o pasaporte del postulante, de su cónyuge o concubino/a y demás miembros mayores de edad del grupo familiar. 3. Casados/as: Certificado de matrimonio original. En caso de existir separación de bienes presentar el documento respaldatorio.

Concubinato: Información sumaria de testigos para acreditar concubinato original expedido por el Poder Judicial o el Juzgado de Paz.

Divorciado/a: Sentencia definitiva o divorcio. Registros Públicos, del postulante, su cónyuge o concubino/a y demás miembros mayores de edad del grupo familiar.

\section{Extranjeros:}

- Radicación permanente.

- Certificado de Interpol vigente expedido por el departamento de Interpol de la Policía Nacional.

- Certificado de antecedentes para extranjeros/as expedido por el departamento de informática de la Policía Nacional

12. En caso de ser repatriado presentar el Certificado de repatriación expedido por el Ministerio de Relaciones Exteriores.

13. Se deben adjuntar, dentro del plazo de presentación de la documentación requerida, un documento respaldatorios emitido por la entidad financiera elegida por el postulante donde conste la conformidad de la misma para otorgar el financiamiento complementario del saldo.

14. Original de la boleta de depósito del Ahorro Previo u Original del extracto actualizado de la caja de ahorro expedido por una IFAC.

15. En caso de solicitar compra de viviendas que no correspondan al MUVH se deben presentar los planos municipales aprobados de la misma. En caso de ser necesario, se aceptaran las contraseñas emitidas por la institución que expide el documento requerido. No obstante, para realizar la evaluación, el documento original en el plazo establecido por el MUVH. Se excluirá toda solicitud que no adjunte íntegramente la documentación exigida, en el plazo de 60 (sesenta) días hábiles, establecidos por el MUVH. 


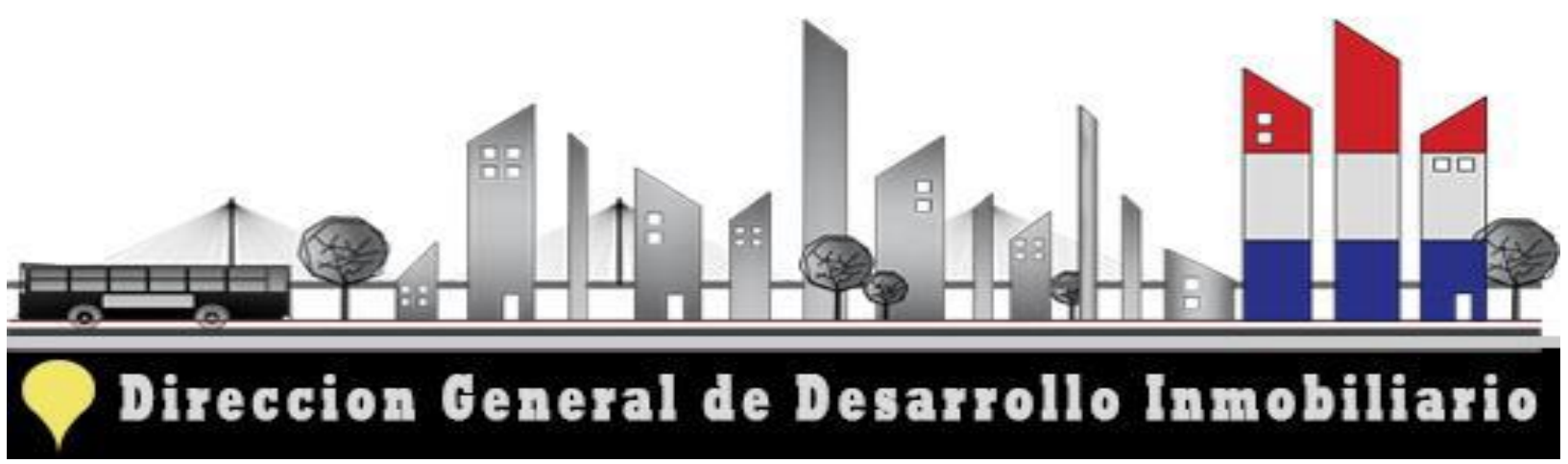

Atención al ciudadano tel.: 0214133000 www.muvh.gov.py email: (Requisitos para compra de Primera Vivienda, n.d.)

Método. Esta investigación se realizó en el marco de una investigación de enfoque cualitativo, de revisión documental, de carácter transversal y alcance descriptivo, que buscó identificar las diversas actividades, procesos y secuencias realizadas en los proyectos de implementación de nuevos requerimientos en la adquisición y/o adjudicación de las nuevas urbes populares y que sea reconocidos y fomentadas en su aplicación con una metodología experimental.

Resultados. Esta investigación cuenta con resultados preliminares en el departamento de Caaguazú, específicamente en el Territorio Social denominado Bonanza de Cnel. Oviedo, donde las viviendas sociales son más llevaderas y con una educación modesta en donde es posible la implementación de proyectos y otros. Claramente se nota la ausencia del interés por la educación que es fundamental para llevar a cabo un buen desarrollo en los territorios sociales. El modelo propuesto debe ser aplicado a nivel nacional a fin de un mejor manejo de los proyectos futuros. 


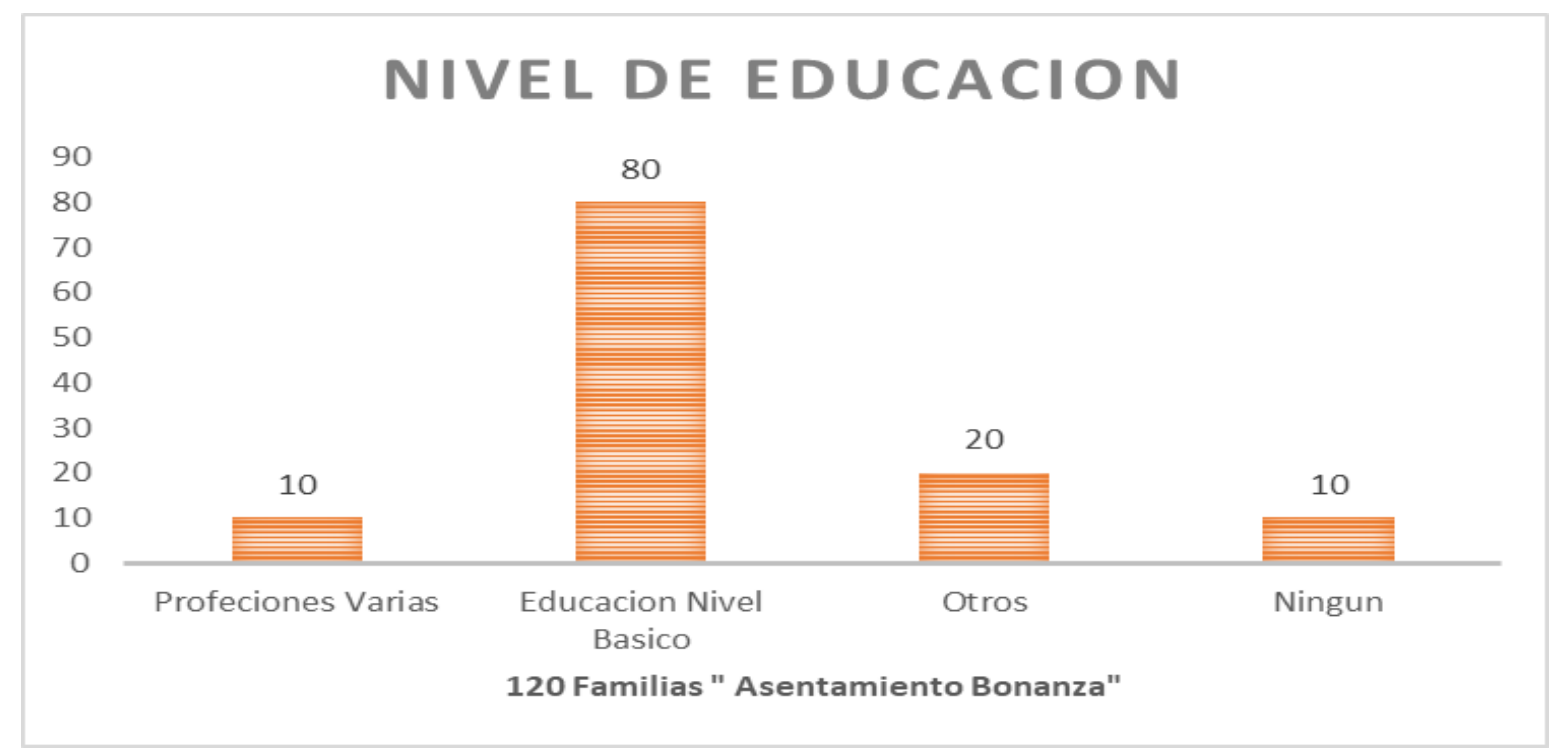

Tabla 1: Grafico de nivel de Educación.

Discusión. El ítem analizado tiene como propósito básico de fomentar una cultura y la innovación en la adjudicación de viviendas populares, a través de procesos de selección de formación en Educación escolar básica y media. Esto implica la nueva ideología que se llevara a cabo, con nuevos enfoques de procesos en el hábitat de las nuevas urbes populares emergentes dentro de todo el territorio paraguayo.

Conclusiones. La implementación de esta propuesta tiene sus frutos en territorios sociales en el Asentamiento Bonanza del departamento de Caaguazú, en donde los habitantes de cada vivienda cuenta con un educación modesta y adecuada para llevar a cabo proyectos establecidos por el gobierno de manera exitosa.

Los requerimientos para el acceso a la vivienda, y con un nuevo ítem propuesto por el investigador mejorará la calidad de vida de sus ocupantes, por ende fomentara la educación e interés de sus habitantes por lo que reduce el índice de dejadez de las mejoras, propuesta, y promoverá el trabajo en equipo, generando nuevas ideas sustentables y sostenibles. 


\section{Bibliografía}

Arrieta, G. M. G. (1999). Acceso a la vivienda y subsidios habitacionales directos : experiencias latinoamericanas.

G, C. F. M. (2014). Diagnóstico Sector Vivienda en Paraguay Índice. Requisitos para compra de Primera Vivienda. (n.d.). 5638.

G, C. F. M. (2014a). Diagnóstico Sector Vivienda en Paraguay Índice.

G, C. F. M. (2014b). Diagnóstico sobre el Fondo Nacional de Vivienda Social de Paraguay Informe Final. 1-52.

Judicial, P. (n.d.). VIVIENDAS ECONÓMICAS.

Manuel Casteel. (2017). Encomia sin Crecimiento (Alianza Ed, p. 303). España.

Precarios, A. (2015). Relevamiento de Asentamientos Precarios.

Marta Isabel Canese. (2019). Urbanizacion Popular en el Area Metropolitana de Asuncion. Asuncion: Dami Creaciones.

Veronica Serafini Geoghegan. (2019). Pobreza en Paraguay: Crecimiento Economico y conflicto redistributivo. Asuncion: QR, impr. desarrollosinmobiliarios@ senavitat.gov.py 


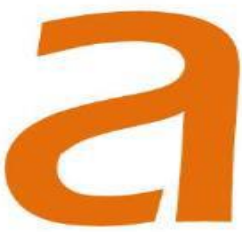

TAS Journal, vol. 4, n. 1, p. 68-77

ISSN: 2595-1521

MARCH 2020

adanibarrola86@gmail.com

\section{ANEXO}

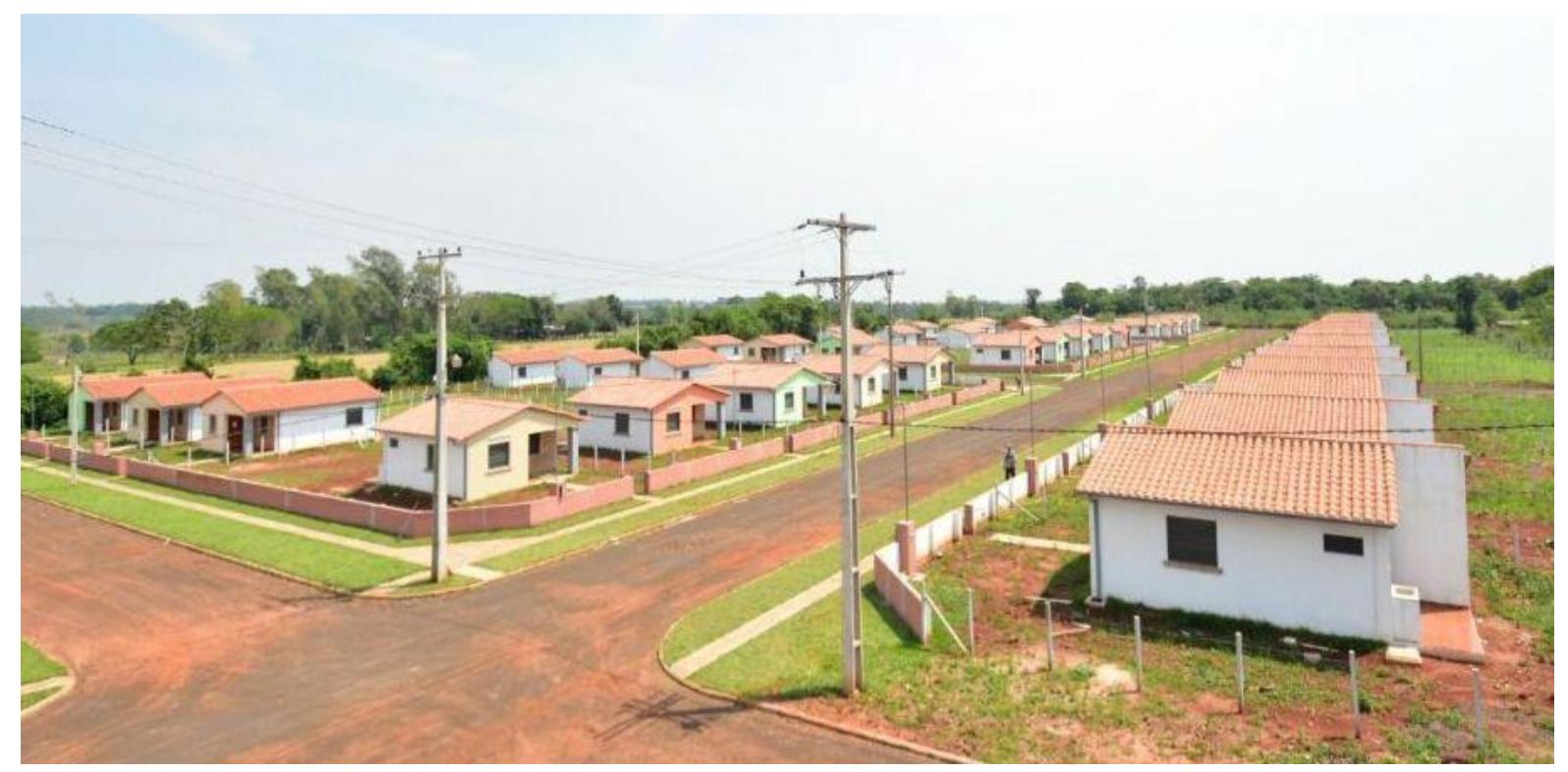

Vivienda Social

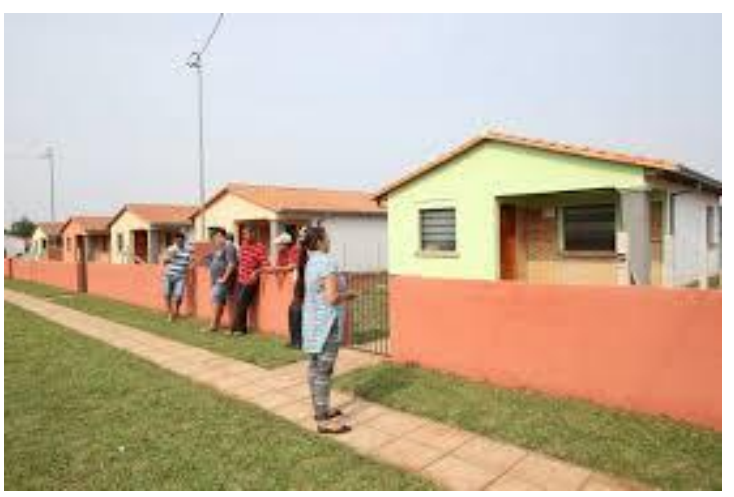

Vivienda Popular

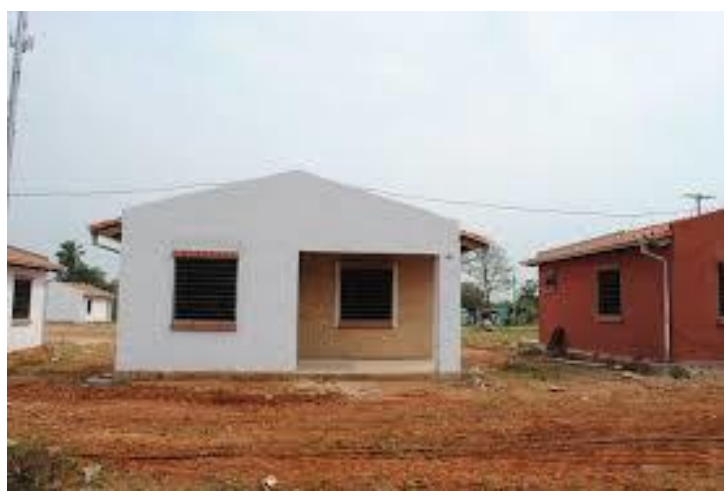

Vivienda Social

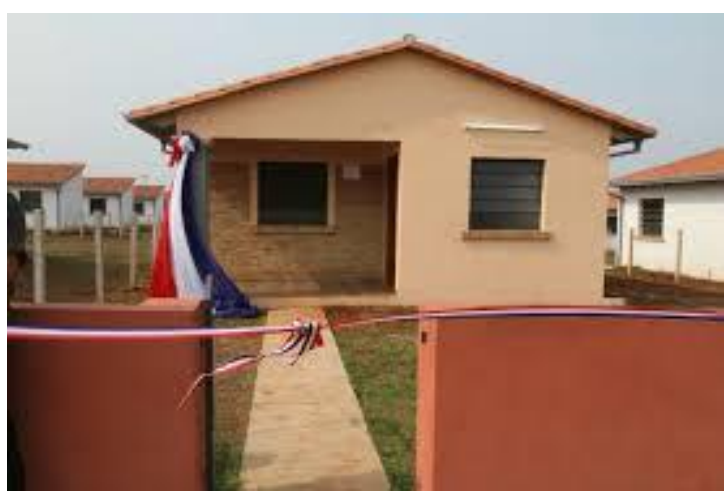

Vivienda social

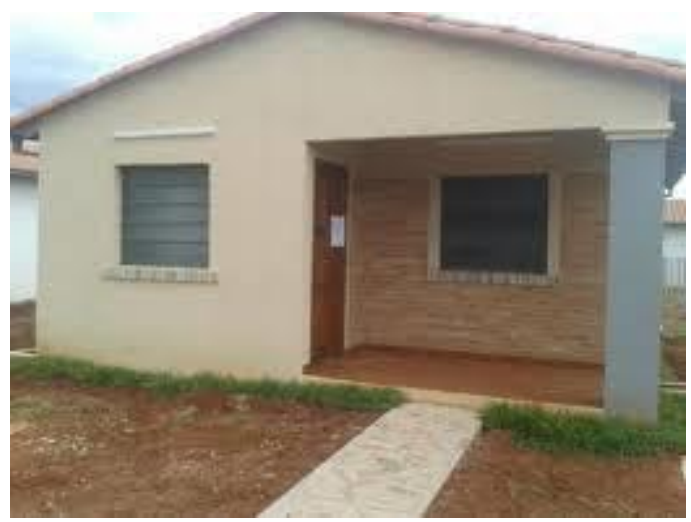

Vivienda Social 